\title{
AVALIAÇÃO NUTRICIONAL SUBJETIVA: Parte 2 - Revisão de suas adaptações e utilizações nas diversas especialidades clínicas
}

\author{
Maria Cristina Gonzalez BARBOSA-SILVA e Aluísio Jardim Dornellas de BARROS
}

RESUMO - Racional - A avaliação nutricional subjetiva é método clínico de avaliação do estado nutricional, que considera não apenas alterações da composição corporal, mas também alterações funcionais do paciente. É processo simples, de baixo custo e não-invasivo, podendo ser realizado à beira do leito. Embora tenha sido desenvolvida para avaliar pacientes cirúrgicos, vários estudos mostram seu uso em outras situações clínicas, como pacientes com insuficiência renal, pacientes oncológicos, hepatopatas, geriátricos e HIV positivos. Objetivo - Revisar o uso da avaliação nutricional subjetiva em outras situações clínicas e estudos de intervenção. Métodos - Foi realizada revisão sistemática no MEDLINE utilizando o descritor "subjective global assessment" e selecionados os trabalhos mais relevantes. Resultados - Foram encontradas adaptações da avaliação nutricional subjetiva para seu uso em pacientes com insuficiência renal, pacientes oncológicos e hepatopatas, com bons resultados neste grupo de pacientes. Em estudos de intervenção em pacientes oncológicos, a avaliação nutricional subjetiva tem sido utilizada para identificar pacientes de maior risco nutricional e que necessitam de terapia nutricional agressiva. Poucos estudos de intervenção de pacientes cirúrgicos utilizaram a avaliação nutricional subjetiva como método para identificação do paciente de risco nutricional. Conclusão - A avaliação nutricional subjetiva tem se mostrado boa opção na avaliação nutricional de pacientes cirúrgicos e algumas modificações têm sido sugeridas para o uso do método também em outras especialidades. A experiência do observador é de extrema importância, uma vez que dela depende a precisão do método.

DESCRITORES - Avaliação nutricional. Transtornos nutricionais.

\section{INTRODUÇÃO}

A avaliação nutricional subjetiva (ANS) é método clínico de avaliação do estado nutricional, desenvolvido por BAKER et al. ${ }^{(2)}$ e DETSKY et al. ${ }^{(9)}$. Diferencia-se dos demais métodos de avaliação nutricional utilizados na prática clínica por englobar não apenas alterações da composição corporal, mas também alterações funcionais do paciente. Trata-se de método simples, de baixo custo e não-invasivo, podendo ser realizado à beira do leito. Apesar de possibilitar a participação de todos os membros da equipe multidisciplinar de terapia nutricional na realização da avaliação nutricional, é indispensável o treinamento adequado de todos os observadores que desejam praticá-la, pois sua precisão depende da capacidade do observador em detectar as alterações nutricionais significativas através da avaliação subjetiva.

Por ser de fácil execução e boa repetibilidade, a ANS vem se tornando o método de escolha também em outras situações clínicas, seja na sua forma original ou após adaptações. Serão discutidos a seguir seu uso e resultado em diversas situações clínicas, assim como estudos de intervenção.

\section{Uso do método em diversas situações clínicas}

Originariamente, a ANS foi desenvolvida e validada para pacientes cirúrgicos ${ }^{(2,8)}$, sendo posteriormente utilizada como método de avaliação nutricional em outros estudos nesses pacientes $^{(13,16,28,29)}$. Em todos estes estudos, exceto um ${ }^{(13)}$, a

Departamento de Medicina Social, Faculdade de Medicina, Universidade Federal de Pelotas, RS, Brasil e Santa Casa de Misericórdia de Pelotas, RS. Trabalho realizado sob patrocínio da Fundação Coordenação de Aperfeiçoamento de Pessoal de Nível Superior - CAPES.

Endereço para correspondência: Dra. Maria Cristina Gonzalez Barbosa e Silva - Rua Ariano de Carvalho, 304 - 96055 -800 - Pelotas, RS. e-mail: mcsilva@epidemio-ufpel.org.br 
ANS conseguiu identificar o grupo de pacientes com maior morbimortalidade.

Vários autores têm relatado seu uso em diversas situações clínicas, sendo que em alguns casos foram sugeridas algumas adaptações do método.

\section{Pacientes nefropatas}

Devido às dificuldades do emprego dos parâmetros nutricionais e antropométricos usualmente utilizados na avaliação nutricional, a ANS tem sido usada como método de avaliação nutricional alternativo neste grupo. Em pacientes com insuficiência renal crônica, foi encontrada correlação entre o diagnóstico de desnutrição realizado pela ANS e métodos objetivos tanto bioquímicos, como a dosagem de transferrina ${ }^{(20)}$, quanto os de composição corporal ${ }^{(12,19)}$. ABDULLAH et al. ${ }^{(1)}$ encontraram menores níveis de fatores anabólicos, como IGF-1 e maiores níveis de citocinas catabólicas em pacientes considerados desnutridos pela ANS, podendo ser esta uma das causas da alta prevalência de sua desnutrição.

Estudos prospectivos realizados em pacientes renais utilizando a ANS mostraram que o método também tem valor prognóstico nesta população. Em pacientes com insuficiência renal aguda, FIACCADORI et al. ${ }^{(14)}$ mostraram que o diagnóstico de desnutrição realizado através da ANS associa-se com maior morbimortalidade e maior custo hospitalar. Em pacientes renais crônicos, a piora do estado nutricional avaliada pela ANS associou-se com aumento de mortalidade em pacientes que realizam diálise peritonial ${ }^{(5)}$, porém não se confirmou como fator que influencie a sobrevida destes pacientes nos estudos de MAIORCA et al. ${ }^{(22,23)}$.

KALANTAR-ZADEH et al. ${ }^{(21)}$ adaptaram a ANS, de forma a torná-la método quantitativo que pudesse ser utilizado em pacientes submetidos a diálise. Cada item é pontuado de acordo com a intensidade da alteração encontrada, variando de normal (1) a muito grave(5). Um "índice de desnutrição" é obtido através da somatória dos pontos obtidos nos 7 itens, sendo que os pacientes normais têm valores baixos (próximo a 7) e os gravemente desnutridos têm valores próximos a 35. Aumento no índice durante a monitorização do paciente seria indicativo de piora no estado nutricional. A ANS quantitativa correlacionou-se significativamente com medidas antropométricas e bioquímicas, necessitando estudos longitudinais para avaliar sua capacidade preditiva de complicações.

\section{Pacientes com neoplasias}

Devido à necessidade de método fácil e de baixo custo que pudesse ser utilizado em pacientes oncológicos ambulatoriais, OTTERY ${ }^{(25)}$ desenvolveu uma forma modificada da ANS denominada avaliação subjetiva global do estado nutricional produzida pelo paciente (ANSPPP). A avaliação consta de questionário, dividido em duas partes, sendo a primeira delas auto-aplicada, com perguntas sobre perda de peso, alteração da ingestão, sintomas (sendo acrescentados alguns relacionados ao paciente oncológico) e alterações na capacidade funcional. A segunda parte do questionário será completada pelo médico, enfermeira ou nutricionista, através da avaliação de fatores associados ao diagnóstico que aumentem a demanda metabólica (como por exemplo: estresse, febre, depressão, fadiga, estádio do tumor ou tratamento), e exame físico semelhante à ANS original. Além da vantagem do paciente sentir-se mais participativo, este método também diminui o tempo gasto pelo profissional para finalizar a avaliação(26) Mostrou-se adequado para identificar pacientes oncológicos que se beneficiariam de intervenção nutricional preventiva durante a terapêutica oncológica ${ }^{(25)}$.

A ANS-PPP pode ser utilizada em diversos tipos de paciente oncológico, havendo boa concordância entre os resultados das avaliações realizadas por médicos e nutricionistas ${ }^{(27)}$. No mesmo estudo, os pacientes oncológicos considerados desnutridos pela ANS-PPP, tiveram níveis significativamente menores de albumina e pré-albumina sérica, assim como menor sobrevida. No entanto, não foram encontradas diferenças significativas entre pacientes considerados desnutridos moderados e graves, sendo questionado se neste grupo esta classificação teria importância clínica.

\section{Pacientes hepatopatas}

Os sinais associados à hepatopatia crônica, tais como ascite, edema, alteração da imunocompetência, diminuição da síntese protéica e insuficiência renal, podem alterar os critérios objetivos tradicionalmente utilizados na avaliação nutricional. Desta forma, a perda de peso, medidas antropométricas, índice creatinina-altura, balanço nitrogenado, excreção de 3-metil-histidina, testes de sensibilidade cutânea, contagem de linfócitos e dosagem sérica de albumina, transferrina, pré-albumina e proteína ligada ao retinol devem ser interpretados com restrições na avaliação do estado nutricional destes pacientes. HASSE et al. ${ }^{(15)}$ introduziram adaptações na ANS original, de forma a torná-la mais adequada a hepatopatas candidatos a transplante hepático. As informações da história são obtidas diretamente do paciente ou de seus familiares, quando existe presença de encefalopatia que possa prejudicar o recordatório. Além dos itens da história e exame físico, um terceiro item completa a avaliação com informações sobre condições mórbidas pré-existentes (presença de encefalopatia, infecções crônicas ou recurrentes, disfunção renal e varizes). Diferentemente da ANS original, o resultado final da avaliação nutricional deve ser baseado nestes três itens conjuntamente. Apesar da pequena população estudada, o método modificado obteve boa concordância entre os observadores, sendo que as perdas das massas musculares e gorduras subcutâneas foram os fatores que mais influenciaram na avaliação nutricional deste pacientes. 


\section{Pacientes geriátricos}

A ANS permite a avaliação nutricional de pacientes geriátricos hospitalizados ou residentes em clínicas geriátricas, pois dispensa maiores recursos técnicos e pode ser realizada à beira do leito ${ }^{(6,11,17)}$. Em pacientes geriátricos hospitalizados, o diagnóstico de desnutrição realizado através da ANS associou-se com maior mortalidade em 90 dias e 1 ano após a alta hospitalar, assim como recuperação funcional mais lenta e maior chance de internações em clínicas geriátricas, sendo estes efeitos independentes da maior gravidade da doença, co-morbidade ou dependência funcional do paciente no momento da internação ${ }^{(6)}$.

BECK et al. ${ }^{(3)}$ realizaram revisão onde analisam os valores de perda de peso significativo e índice de massa corporal adequado para pacientes idosos. Segundo estes autores, perda de peso de $5 \%$ em 1 ano já é clinicamente significativa neste grupo, sendo sugerido que, para a interpretação da perda de peso em 6 meses na ANS, qualquer valor seja considerado significativo.

\section{Pacientes HIV positivos}

A ANS tem sido utilizada também na avaliação nutricional de pacientes HIV positivos. O método torna-se útil principalmente em pacientes ambulatoriais ${ }^{(24)}$, já que pode ser realizado por profissionais não-médicos, como enfermeiras e nutricionistas ${ }^{(4)}$. Também nesta população a ANS associou-se significativamente com outros métodos objetivos de avaliação nutricional. Pacientes HIV positivos considerados desnutridos graves pela ANS, apresentaram valores significativamente menores de albumina sérica e contagem de linfócitos CD4 ${ }^{(4)}$ e alterações na composição corporal ${ }^{(24)}$.

Em estudos prospectivos, a ANS mostrou-se útil no acompanhamento de pacientes ambulatoriais infectados com o HIV. Os pacientes que apresentaram piora no estado nutricional segundo a ANS, correlacionaram-se significativamente com a piora na classificação CDC para a doença ${ }^{(24)}$, demonstrando a sensibilidade do método nesta situação clínica. Neste estudo, porém, a desnutrição diagnosticada pela ANS foi subestimada quando comparada com a perda de peso isoladamente como critério diagnóstico.

\section{Intervenções}

A ANS parece ser capaz de identificar adequadamente os pacientes de maior risco de apresentar complicações pós-operatórias ou em situações clínicas, e considera-se que estas complicações sejam mediadas pelas alterações funcionais decorrentes da desnutrição. Segundo este raciocínio, a intervenção terapêutica - neste caso, terapia nutricional - deveria ser capaz de reverter o maior risco encontrado em pacientes desnutridos graves. No entanto, alguns aspectos devem ser discutidos. Em primeiro lugar, existe a possibilidade de que a ANS possa ser marcador para um "estado de saúde/doença", que vai além da definição de desnutrição, decorrente da ingestão inadequada de calorias, proteínas e micronutrientes ${ }^{(10,18)}$. Sob este raciocínio, a repleção de macro e micronutrientes somente não seria capaz de modificar o risco nutricional associado aos pacientes considerados desnutridos graves. Outra possibilidade seria a desnutrição, como diagnosticada pela ANS, um fenômeno contínuo, apenas iniciado pela ingestão insuficiente de macro e micronutrientes, progredindo a partir de alterações funcionais que precedem as alterações da composição corporal e tais alterações seriam responsáveis pelo maior risco de complicações ${ }^{(18)}$. Sendo assim, apenas a restauração da função poderia diminuir o risco nutricional destes pacientes.

Deve-se considerar, também, a via pela qual será realizada a intervenção nutricional, uma vez que o próprio método pode estar associado à presença de complicações, como no caso da nutrição parenteral. O estudo multicêntrico coordenado por Buzby ${ }^{(29)}$, mostrou pela primeira vez que parte dos resultados da terapia nutricional depende da via que se utiliza, parenteral ou enteral. Nesse ensaio clínico e aleatório, inúmeras considerações poderiam ser feitas, tais como: pequeno número de pacientes que efetivamente participaram do estudo (395 pacientes entre 3.259 potencialmente elegíveis) e ao próprio método de intervenção utilizado (nutrição parenteral mesmo com trato digestivo disponível e excesso de calorias ofertadas). Nesse estudo não foi observada boa concordância entre os métodos objetivos e subjetivos, uma vez que após serem selecionados para o estudo, critérios objetivos (\% peso ideal, albumina, pré-albumina ou índice de risco nutricional (INR)), classificados pela ANS, mostrou que $30 \%$ dos pacientes que foram incluídos na aleatoriedade não apresentavam déficit nutricional pela avaliação subjetiva. No grupo controle tanto a classificação subjetiva (ANS), como a objetiva (INR) sugere maior incidência de complicações conforme a piora da gravidade da desnutrição, porém esta associação não foi estatisticamente significativa. No grupo que recebeu nutrição parenteral total, os pacientes identificados como desnutridos moderados, tiveram aumento significativo das complicações infecciosas, e apenas aqueles considerados desnutridos graves pela ANS tiveram benefício sugerido da intervenção nutricional na forma de nutrição parenteral. Este benefício se resumiu a menor incidência de complicações maiores (infecciosas e não-infecciosas) (25,8\%), comparando-se com o grupo controle $(47,4 \%)$. A redução de complicações observada não foi estatisticamente significativa, sendo que o limitado número de pacientes neste subgrupo certamente comprometeu o poder do estudo.

DETSKY et al. ${ }^{(7)}$ já haviam considerado anteriormente que a via utilizada para a intervenção nutricional interfere na decisão de se tratar todos, alguns ou nenhum paciente submetido a cirurgia do trato gastrointestinal. Pela análise desses autores, a decisão deve ser tomada levando-se em consideração não apenas as propriedades preditivas do método diagnóstico utilizado, mas também as complicações iatrogênicas inerentes ao método de intervenção nutricional.

Em pacientes oncológicos a utilização da ANS para identificar os que se beneficiariam da intervenção nutricional, tem tido bons 
resultados ${ }^{(25)}$. Um algoritmo é utilizado, baseando-se na classificação do estado nutricional segundo versão modificada da ANS e no risco nutricional que a terapia oncológica a ser aplicada oferece (considerada de baixo ou alto risco para o aparecimento de sintomas de impacto nutricional, tais como náusea, vômitos, diarréia, etc). Segundo o algoritmo, os pacientes são encaminhados para receber apenas material educativo geral, orientação nutricional específica ou intervenção nutricional, que pode variar desde suplementação oral, a terapia nutricional enteral ou parenteral preventiva, se necessário. Desta maneira, tem-se obtido a manutenção ou melhora do peso e das proteínas viscerais durante a terapia oncológica.

Em resumo, são poucos os estudos de intervenção nutricional que utilizaram a ANS como método de avaliação inicial de critério de elegibilidade na identificação do paciente de risco nutricional. O maior estudo realizado utilizou o método não como critério de elegibilidade e os resultados obtidos não podem ser conclusivos devido a vários problemas metodológicos do estudo. No entanto, em pacientes oncológicos, a ANS tem sido útil como método de identificação de pacientes com maior risco nutricional e potenciais candidatos a uma intervenção nutricional precoce e preventiva.

\section{CONCLUSÕES}

Apesar de originariamente desenvolvido para uso em pacientes cirúrgicos, a ANS vem se tornando o método de escolha também em outras situações clínicas, seja na sua forma original ou após adaptações.

A ANS tem sido utilizada também em outras áreas clínicas, além do paciente cirúrgico. Pequenas modificações têm sido sugeridas para individualizar o método para cada particularidade da situação clínica em questão. Desta maneira, tem se comprovado a idéia de que a boa avaliação clínica realizada por profissional atento para as modificações que a desnutrição pode ocasionar, consegue identificar os pacientes de maior risco nutricional nas diversas situações clínicas, seja ele portador de neoplasia ou seja renal crônico.

Mais estudos de intervenção, utilizando o método como diagnóstico do risco nutricional tornam-se necessários para sua melhor avaliação. As evidências de que o método consegue identificar os pacientes que realmente poderiam beneficiar-se de uma terapia nutricional ainda não são suficientes para que seu uso seja universalmente utilizado como avaliação inicial no paciente hospitalizado. $\mathrm{O}$ fato de identificar os pacientes de maior risco para morbimortalidade sem a certeza de meios de revertê-lo, torna a avaliação de risco nutricional de pouco valor prático no manuseio clínico do paciente.

A complementação da ANS com outras técnicas de avaliação nutricional é sugerida, uma vez que o método não possui sensibilidade adequada para identificar pequenas variações no estado nutricional e, portanto, não é indicado como método de acompanhamento da evolução do paciente em terapia nutricional.

Barbosa-Silva MCG, Barros AJD. Subjective global assessment. Part 2. Review of its adaptations and utilization in different clinical specialties. Arq Gastroenterol 2002;39(4):248-252.

ABSTRACT - Background - The subjective global assessment is a clinical method for nutritional assessment that considers not only body composition alterations but also changes in physiological function. The method is simple, inexpensive and non-invasive, and it can be performed at bedside. Although the subjective global assessment was developed to assess surgical patients, many studies have showed its use in other clinical situations, evaluating patients with renal failure patients, cancer, liver diseases, as well as elderly and HIV-infected patients. Aim - To review the use of subjective global assessment in other clinical situations and intervention studies. Methods - It was performed a systematic review in MEDLINE, using subjective global assessment as search term and the most relevant papers were selected. Results - It was found the subjective global assessment modifications to be applied in renal failure patients, cancer and liver diseases, with good results. In patients with cancer, the subjective global assessment has been used to identify patients who are at higher nutritional risk and would need an aggressive nutritional support. Few intervention studies in surgical patients used subjective global assessment to identify nutritional risk patients. Conclusions - The subjective global assessment has been a good option in nutritional assessment in surgical patients and some modifications have been suggested to adjust the method to other clinical situations. The observer experience is of extreme importance, since the precision of the method depends on it.

HEADINGS - Nutrition assessment. Nutrition disorders

\section{REFERÊNCIAS BIBLIOGRÁFICAS}

1. Abdullah MS, Wild G, Jacob V, Milford-Ward A, Ryad R, Zanaty M, Ali MH, Nahas AME. Cytocines and the malnutrition of chronic renal failure. Miner Electrolyte Metab 1997;23:237-42.

2. Baker JP, Detsky AS, Wesson DE, Wolman SL, Stewart S, Whitewell J, Langer B, Jeejeebhoy KN. Nutritional assessment: a comparison of clinical judgment and objective measurements. N Engl J Med 1982;306:967-72.
3. Beck AM, Ovesen L. At which body mass index and degree of weight loss should hospitalized elderly patients be considered at nutritional risk? Clin Nutr $1998 ; 17: 195-8$

4. Bowers JM, Dols CL. Subjective global assessment in HIV-infected patients. J Assoc Nurses AIDS Care 1996;7:83-9.

5. Canada-USA (CANUSA) Peritoneal Dialysis Study Group. Adequacy of dialysis and nutrition in continous peritoneal dialysis: association with clinical outcomes. J Am Soc Nephrol 1996;7:198-207. 
6. Covinsky KE, Martin GE, Beyth RJ, Justice AC, Sehgal AR, Landefeld CS. The relationship between clinical assessments of nutritional status and adverse outcomes in older hospitalized medical patients. J Am Geriatr Soc 1999;47:532-8.

7. Detsky AS, Mendelson RA, Baker JP, Jeejeebhoy KN. The choice to treat all, some, or no patients undergoing gastrointestinal surgery with nutritional support: a decision analysis approach. JPEN J Parenter Enteral Nutr 1984;8:245-53.

8. Detsky AS, Baker JP, O'Rourke K, Johnston N, Whitwell J, Mendelson RA, Jeejeebhoy KN. Predicting nutrition-associated complications for patients undergoing gastrointestinal surgery. JPEN J Parenter Enteral Nutr 1987;11:440-6

9. Detsky AS, McLaughlin JR, Baker JP, Johnston N, Whittaker S, Mendelson RA, Jeejeebhoy KN. What is subjective global assessment of nutritional status? JPEN J Parenter Enteral Nutr 1987:11:8-13.

10. Detsky AS. Nutritional status assessment: does it improve diagnostic or prognostic information? Nutrition 1991;7:37-8.

11. Ek A-C, Unosson M, Larsson J, Ganowiak W, Bjurulf P. Interrater variability and validity in subjective global assessment of elderly patients. Scan J Caring Sci $1996 ; 10: 163-8$

12. Enia G, Sicuso C, Alati G, Zoccali C. Subjective global assessment of nutrition in dialysis patients. Nephrol Dial Transplant 1993;8:1094-8.

13. Faintuch J, Cohen RV, Machado MCC, Pinotti HW. Avaliação nutricional subjetiva de pacientes cirúrgicos com câncer. Rev Paul Med 1988;106:155-60.

14. Fiaccadori E, Lombardi M, Leonardi S, Rotelli CF, Tortorella G, Borghetti A. Prevalence and clinical outcome associated with preexisting malnutrition in acute renal failure: a prospective cohort study. J Am Soc Nephrol 1999;10:58193.

15. Hasse J, Strong S, Gorman MA, Liepa G. Subjective global assessment: alternative nutrition-assessment technique for liver-transplant candidates. Nutrition 1993;9:339-43.

16. Hirsch S, de Obaldia N, Petermann M, Rojo P, Barrientos C, Iturriaga H, Bunout D. Subjective global assessment of nutritional status: further validation. Nutrition 1991;7:35-8.

17. Irving GF, Olsson BA, Cederholm T. Nutritional and cognitive status in elderly subjects living in service flats, and the effect of nutrition education on personnel. Gerontology 1999;45:187-94.

18. Jeejeebhoy KN, Detsky AS, Baker JP. Assessment of nutritional status. JPEN J Parenter Enteral Nutr 1990;14:193S-6S.
19. Jones CH, Newstead CG, Will EJ, Smye SW, Davison AM. Assesment of nutritional status in CAPD patients: serum albumin is not a useful measure. Nephrol Dial Transplant 1997; 12:1406-13

20. Kalantar-Zadeh K, Kleiner M, Dunne E, Ahern K, Nelson M, Koslowe R, Luft FC. Total iron-binding capacity-estimated transferrin correlates with the nutritional subjective global assessment in hemodialysis patients. Am J Kidney Dis 1998;31:263-72.

21. Kalantar-Zadeh K, Kleiner M, Dunne E, Lee GH, Luft FC. A modified quantitative subjective global assessment of nutrition for dialysis patients. Nephrol Dia Transplant 1999;14:1732-8

22. Maiorca R, Brunori G, Zubani R, Cancarini GC, Manili L, Camerini C, Movilli E, Pola A, d'Avolio G, Gelatti U. Predictive value of dialysis adequacy and nutritional indices for mortality and morbidity in CAPD and HD patients. A longitudinal study. Nephrol Dial Transplant 1995;10:2295-305.

23. Maiorca R, Cancarini GC, Brunori G, Zubani R, Camerini C, Manili L, Movilli E. Comparison of long-term survival between hemodialysis and peritoneal dialysis Adv Perit Dial 1996;12:79-88

24. Niyongabo T, Melchior JC, Henzel D, Bouchaud O, Larouze B. Comparison of methods for assessing nutritional status in HIV-infected adults. Nutrition 1999;15:740-3.

25. Ottery FD. Definition of standardized nutritional assessment and interventional pathways in oncology. Nutrition 1996;12:S15-S19.

26. Ottery FD. Nutritional oncology: a proactive, integrated approach to the cancer patient. In: Shikora SA, Blackburn GL, editors. Nutrition support: theory and therapeutics. New York: Chapman \& Hall; 1997. p. 395-409.

27. Persson C, Sjoden PO, Glimelius B. The Swedish version of the patient-generated subjective global assessment of nutritional status: gastrointestinal vs urological cancers. Clin Nutr 1999;18:71-7.

28. Sánchez Arias M, Argüello Choisoul A, Fallas ML, Rojas J, Quesada Esquivel R. Aplicación de la valoración global subjetiva nutricional en un hospital clase A. Rev Méd Costa Rica Centroam 1999;66(546):23-5.

29. The Veterans Affairs Total Parenteral Nutrition Cooperative Group. Perioperative total parenteral nutrition in surgical patients. N Engl J Med 1991;325:525-32.

Recebido em 3/9/2001 Aprovado em 6/3/2002 